\title{
Chapitre 8
}

\section{Martin Brendel}

Martin Brendel (1862-1939) a fait ses études de mathématiques et d'astronomie à Berlin, Munich, Stockholm, Paris et Londres. Il a soutenu sa thèse sur la méthode de Gyldén à Berlin (Brendel 1890), et il a obtenu son habilitation à Griefswald en 1892. En 1898, il fut nommé professeur assistant d'astronomie théorique à l'Université de Göttingen, où il a eu l'occasion d'enseigner les mathématiques d'assurance-vie et les statistiques mathématiques, ainsi que l'astronomie théorique. En 1907, il est devenu professeur d'astronomie et de mathématiques d'assurance-vie à l'Akademie für Sozial- und Handelswissenschaften à Frankfurt a. M., et l'année suivante, directeur du nouvel observatoire dans cette même ville. À propos de la vie et les travaux de Brendel, voir Boda (1940).

\subsection{Poincaré à Brendel}

$[24.01 .1912]^{1}$

Mon cher Collègue,

Je suis bien négligent; voilà déjà plusieurs semaines que le Bureau des Longitudes m'avait chargé de vous écrire que nous serions heureux d'envoyer à Francfort de jeunes astronomes français afin de s'initier, tout en vous aidant à vos travaux, à la théorie des petites planètes. ${ }^{2} \mathrm{Il}$ y a toutefois encore quelques difficultés que nous espérons surmonter.

Votre bien dévoué,

Poincaré

ALS 1p. Acc. Darms. 9.8.28, Handschriftenabteilung, Staatsbibliothek Berlin.

1. Date de réception, selon une annotation de main inconnue : "24.1.12."

2. Un jeune astronome français, Jean Trousset (1885-1943), a collaboré avec Brendel en 1913-1914, selon son exposé de la méthode de calcul d'orbites de petites planètes de Brendel (Trousset (1933). Par la suite, Trousset est devenu professeur de mécanique rationnelle à la Faculté des sciences de Bordeaux, et doyen (Véron 2006). 